\title{
CovID-19 Teleophthalmology and COVID-19: the patient perspective
}

\author{
Authors: Vidushi Golash, ${ }^{A}$ Sarju Athwal ${ }^{B}$ and Mona Khandwala ${ }^{B}$
}

\begin{abstract}
Oculoplastic services at a UK district general hospital underwent reconfiguration to incorporate teleconsultations during the COVID-19 pandemic, and patient satisfaction was assessed.
\end{abstract}

\section{Methods}

All oculoplastic patients at Maidstone Hospital underwent telephone or video consultations in place of face-to-face reviews. Patient feedback surveys were conducted.

Results

80 telephone and 40 video consultation responses were analysed. The majority of teleconsultations lasted 6-10 minutes. $55 \%$ of telephone and $82.5 \%$ of video consultation patients felt face-to-face reviews would not have changed the appointment outcome. Satisfaction scores of 10/10 were given by $71.3 \%$ of telephone and $72.5 \%$ of video consultation patients. Correlation between age and preference of consultation type was observed, with $62.5 \%$ of patients aged $>65$ years requesting regular face-to-face reviews compared to only $18.8 \%$ of $25-64$-year-olds.

Conclusion

Patients highly support teleconsultation adaptations. This is an opportunity to incorporate and enhance teleconsultation facilities to meet current and future demand, especially with ongoing social distancing guidelines.

KEYWORDS: ophthalmology, teleophthalmology, COVID-19, quality improvement, telemedicine

DOI: $10.7861 /$ fhj.2020-0139

\section{Introduction}

A key aim of the NHS England Long Term Plan is the reduction of up to one third of outpatient visits over the next 5 years by incorporating digital services into the existing secondary care outpatient framework.' Telephone and video consultations have been in progress over the last few years across the UK. ${ }^{2-4}$ However,

Authors: AST2 ophthalmology, Maidstone Hospital, Maidstone, UK; ${ }^{B}$ Consultant ophthalmologist, Maidstone Hospital, Maidstone, UK previous studies have demonstrated adaptation is often slow and disruptive to existing frameworks. ${ }^{5,6}$

The ongoing COVID-19 pandemic has accelerated the adoption of digital services at an unprecedented rate, described by some as '10 years of change in one week'? The highly contagious nature of this virus has necessitated policies of self-isolation and social distancing. Although sight-threatening conditions are still being prioritised and treated appropriately, ${ }^{8}$ much elective work has been postponed, with some reports stating that ophthalmology outpatient visits have reduced by up to $79 \%$.

The contagious nature of the virus and the close nature of the eye examination are of particular concern in ophthalmology. By replacing face-to-face consultations with teleophthalmology, we can reduce individual exposure in the hospital environment and time spent in close proximity, thus protecting both patients and clinicians.

While there is an important role for telephone consultations within ophthalmology, the highly visual nature of the specialty makes it very suitable for video consultations. Oculoplastics in particular, being a macroscopic subspecialty, offers great opportunity to encompass video consultations with the existing outpatient framework..$^{10}$ Many eyelid and lacrimal diseases can often be easily visualised and examined without the use of a slit lamp."11

A number of video consultation platforms have been approved by NHS Digital, ${ }^{12}$ including the Attend Anywhere ${ }^{\circledR}$ platform (Attend Anywhere; Melbourne, Australia), which has been procured by NHS England and NHS Improvement as part of their secondary care support measures. ${ }^{13}$ There are already reports that Attend Anywhere has improved care pathways within ophthalmology. ${ }^{14,15}$

Although these reports demonstrate technical feasibility, a large hurdle to widespread adoption of teleophthalmology will be user acceptance. Analysis of patient interactions with the online consultation system askmyGP (askmyGP; Cossington, UK) has shown that the majority of patients requested telephone consultations rather than video. ${ }^{16}$ This is of particular importance in ophthalmology, given the large proportion of older patients. A recent survey showed that patients over the age of 70 may avoid a GP appointment if it is made by video call. ${ }^{17}$

We describe our experiences of establishing teleophthalmology services within our existing framework at Maidstone and Tunbridge Wells NHS Trust, and describe patient-reported outcomes following teleophthalmology oculoplastic clinics as part of the COVID-19 response strategy. 


\section{Methods}

As part of the pre-lockdown social distancing response, a Trust-wide decision was made to convert all booked outpatient appointments to telephone consultations from 23 March 2020, with a plan to arrange face-to-face appointments for those patients needing urgent review. For the first 2 weeks, all consultations were conducted on-site and via telephone.

With the help of our transformation programme manager, we were rapidly able to acquire webcams for all desktop computers normally used by the oculoplastic team. We also gained access to the Attend Anywhere video consultation platform and were therefore able to commence video consultations from 9 April 2020. When this infrastructure was in place, patients were given a choice between telephone or video consultation (as time and clinic capacity allowed). To do this, a handful of randomly selected patients per clinic list per week were directly phoned by the oculoplastics administrative assistant to ask if they would be able to attend a video consultation. The only requirement for participation was access to an internet-connected device equipped with a video camera (eg a webcam or a built-in camera on a laptop or smartphone). Both new and follow-up patients were included. Those who consented to video consultation provided an email address to be securely stored on their patient record. An appointments booking clerk then emailed a template of simple instructions, including a video consultation link and the patient's appointment date and time, to the patient's email address. To better understand patient preferences, we kept a secure record of the patients contacted during a 1-month period, including demographic data and their preferred consultation type.

\section{Survey}

A 20-question telephone consultation survey and 22-question video consultation survey was designed by the authors using Google Forms (Google; California, USA) and SurveyMonkey@ for Business (SurveyMonkey; California, USA) respectively (see supplementary material S1 and S2). The surveys were conducted by ophthalmic administrative assistants via telephone within 24 hours of the consultation, with responses recorded electronically. The survey administrators randomly chose three patients from each clinic list in the 8-week period from 26 March to 21 May 2020 to ask if they would be willing to take part in the survey. Both new and follow up patients were included. Main recorded measures were patient demographics, comparison with any previous face-to-face consultations, elements of convenience, perceived clinical outcome and overall satisfaction. Questions were a mixture of yes/no and single answer. Questions about convenience and satisfaction were based on a 10-point Likert scale.

\section{Results}

Between the specified dates, 209 telephone consultations and 50 video consultations were conducted in the oculoplastics outpatient clinics. 80 telephone consultation surveys and 40 video consultation surveys were completed. Results of each have been discussed separately below. The demographic spread of survey participants is shown in Table 1.

Of the 120 survey respondents, 90 (75\%) were follow-up patients, the majority of whom (81\%) previously travelled to their
Table 1. Patient demographics of those who provided

feedback following teleconsultations

\begin{tabular}{llll}
\multirow{3}{*}{ Gender } & Male & $\begin{array}{l}\text { Video } \\
(\mathbf{n}=\mathbf{4 0})\end{array}$ & $\begin{array}{l}\text { Telephone } \\
(\mathbf{n}=\mathbf{8 0})\end{array}$ \\
& Female & 23 & 37 \\
Age (years) & Unanswered & 0 & 41 \\
& Under 18 & 4 & 2 \\
& $18-24$ & 0 & 3 \\
& $25-35$ & 4 & 0 \\
& $35-44$ & 1 & 2 \\
& $45-54$ & 6 & 0 \\
& $55-64$ & 9 & 4 \\
& $65-74$ & 12 & 10 \\
& $75-84$ & 4 & 22 \\
& $85+$ & 0 & 30 \\
& Unanswered & 0 & 5
\end{tabular}

clinic appointments by car. Fig 1a shows the journey duration patients endured to travel to their previous clinic appointments. Over a third (34.2\%) of all participants were also dependent on someone else to bring them to their face-to-face hospital appointment.

\section{Telephone consultation: patient-reported outcomes}

When asked about their telephone consultation appointment, $35 \%$ of all participants recalled being provided with an appointment date and time. A further third (33.8\%) felt they had only been given an appointment date, whereas the remaining felt they were not given any prior appointment details. Despite this varied feedback, $97.2 \%$ of all respondents did not experience any delay with the telephone consultation, or any inconvenience if there was a delay.

Regarding the telephone consultation itself, all but three calls took less than 15 minutes (Fig 1b). 100\% of respondents felt they were listened to by their doctor and had enough time to discuss their individual situation, including any changes to treatment. Overall satisfaction and convenience scores out of 10 were requested from patients with regards to their opinion on their telephone consultation. $71.3 \%$ of patients gave a full $10 / 10$ score for overall satisfaction, and $78.8 \%$ of all replies voted $10 / 10$ for convenience (Fig 2).

Despite this high level of satisfaction and convenience associated telephone consultations, when asked to give a direct preference between telephone and face-to-face consultation types, patient preferences were found to correlate with patient age. $70.9 \%$ of respondents ( 56 out of 75 who provided data on age) were 65 years or older, and $62.5 \%$ of this older cohort would choose face-to-face consultations every time, much like the prepandemic clinic style. In contrast, only three of the 16 participants aged $25-64$ years (18.8\%) would want in-person reviews every time, with the remaining majority welcoming telephone consultations where relevant and possible. 
a

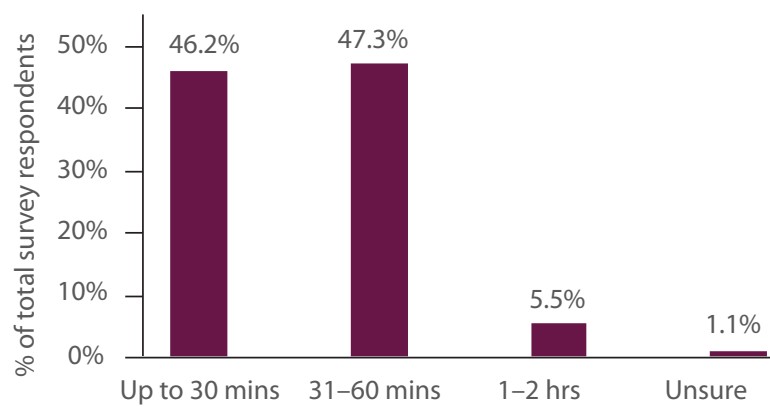

b

Duration in minutes

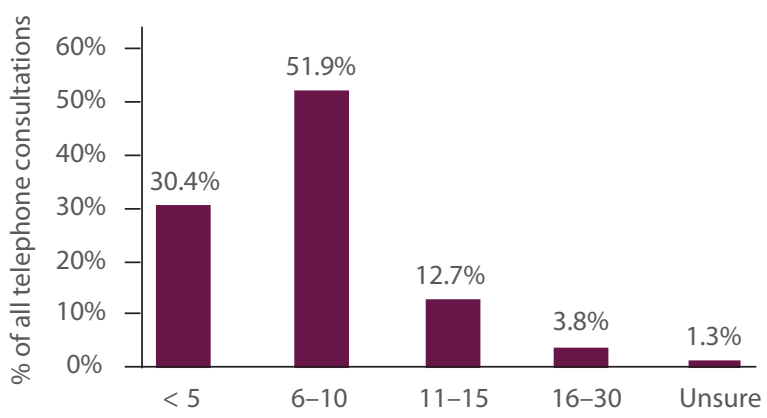

C

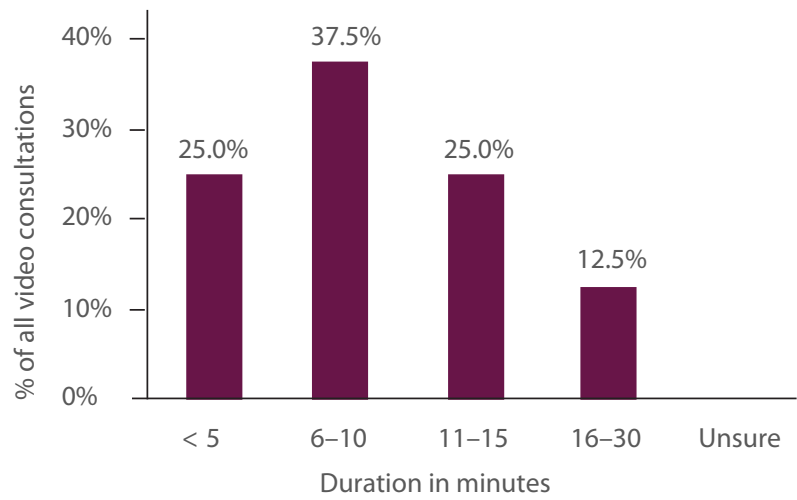

Fig 1. Percentage distribution of a) journey times to hospital for all follow-up patients, b) duration of telephone consultations and c) duration of video consultations.

Lastly, patients were asked if they felt a face-to-face consultation would have changed the outcome of their appointment. Just over half of those asked (55\%) replied no, and the remainder felt there may have been some difference (Fig 3).

\section{Video consultation: patient-reported outcomes}

We assessed the uptake rate of video consultation appointments (VCAs) when patients were directly offered a choice between telephone or video consultations (Fig 4). Over a 1-month period, 89 oculoplastic patients were directly phoned by a third-party administrative clerk. Of those who we could reach, $84 \%$ (47 of 56) opted for a video consultation. Only nine individuals declined, with the most common reasons given being limited access to smartphone technology or patient preference.

The majority of VCA patients $(87.5 \%)$ recalled being given an appointment date and time. $80 \%$ of VCA patients reported starting their appointment on time or promptly within 15 minutes. This is a significant contrast to the previous in-person appointments; of all the follow-up patients participating in the survey, only $35 \%$ felt they had been seen within 15 minutes of their most recent in-person clinic appointment time.

The average video consultation duration was 13 minutes, ranging from 1 minute for an uncomplicated, satisfied postoperative review, to a maximum of 20 minutes for a new referral for thyroid eye disease (Fig 1c). 100\% of survey participants felt they had enough time for discussion with their doctor and ask questions where needed. This observed pattern of video consultation duration, corresponding with high-level patient satisfaction regarding length of appointment, allows us to keep our current clinic template the same: 15-minute appointments per patient (both new and follow-up), and 30-minute appointments for thyroid eye disease patients.

Our survey also sought to assess patient perceived convenience and difficulties regarding the technical aspects of video consultations. To prepare for their VCA, patients were sent an email template with instructions and personalised appointment information. Survey participants were asked to report a difficulty score out of 10 ( 1 = very easy, $10=$ very difficult $)$ with regards to ease of understanding the given information. The average reported difficulty score was 2 (ie 'very easy'). When asked if there were any further details that would make the preparation for video consultation easier, $92 \%$ of patients reported 'no further details needed'. The few free-text suggestions provided were: bolder formatting on the email to highlight the video consultation link; prior notice on the Attend Anywhere platform before the patient proceeds to the virtual waiting room; and an animation/video to familiarise with Attend Anywhere prior to the appointment. Regarding the consultation itself, $57.5 \%$ of participants experienced no technical problems. The most common difficulty (20\%) was problems with audio, followed by issues with the Attend Anywhere platform (10\%). Only a couple of individuals reported inconveniences related to internet connections or personal equipment (eg camera facing the wrong direction, poor light etc). Much like the telephone survey, despite any technical difficulties, a significant majority of video consultation patients went on to report overall convenience and satisfaction scores of 10/10 (Fig 2). The most commonly reported advantages of VCA were saving time travelling to and waiting in hospital, saving money by not needing to travel or park and having a generally less stressful experience than when attending for in-person reviews. Patients with medical comorbidities or limited mobility also reported the enhanced ease and safety of a consultation from home. Patients appreciated not needing to take time off work or arrange childcare/care for a relative if these applied. Regarding disadvantages, 10 people reported mild to moderate technical difficulties. Only two individuals felt the process was too complicated, and a further two struggled to find a suitable location for their video consultation.

Lastly, we sought to establish patient perception regarding the outcome of the consultation. $82.5 \%$ of patients who had a VCA felt a face-to-face review would not have changed the outcome of their consultation. This is significantly higher than a corresponding 


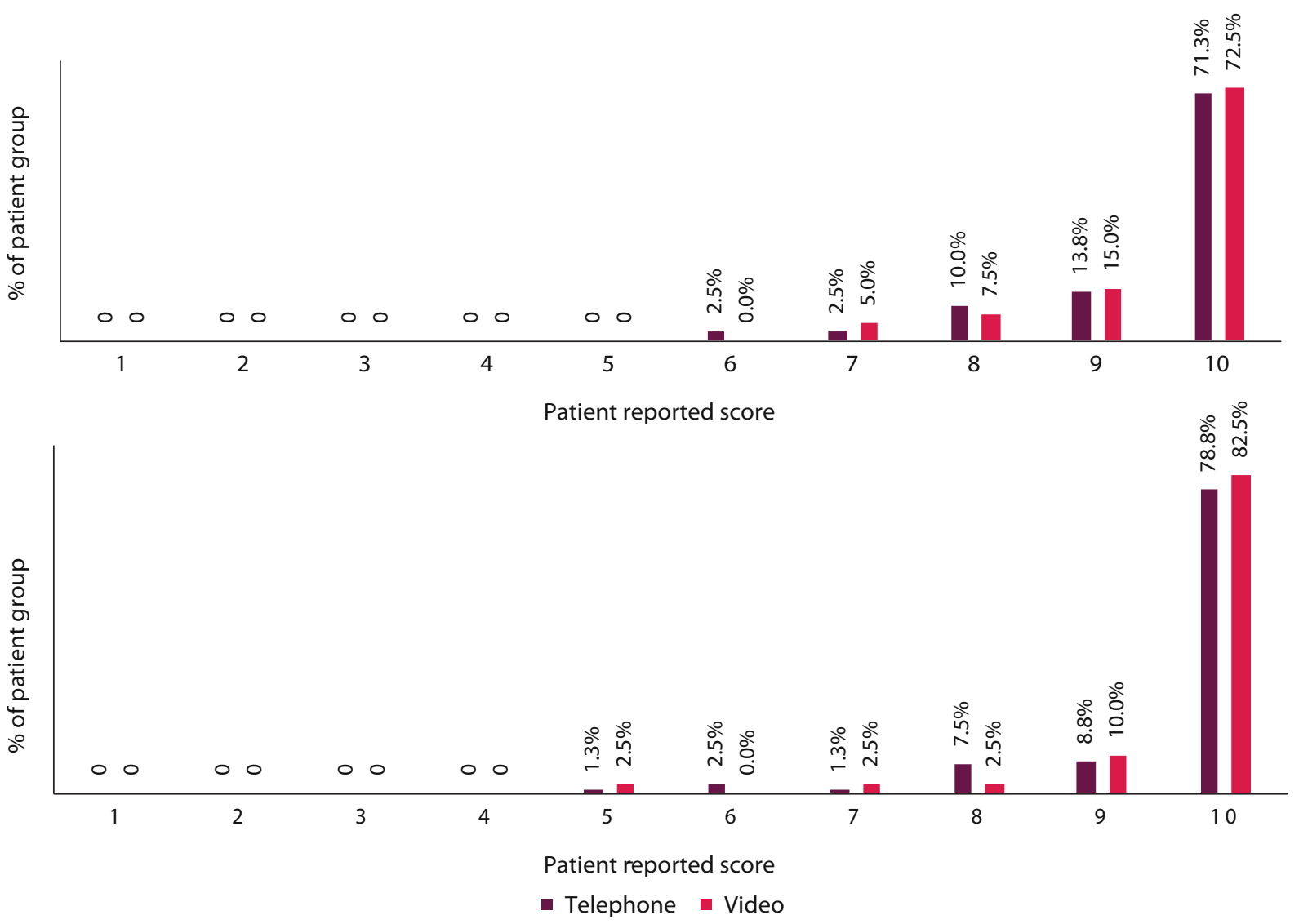

Fig 2. Patient-reported overall satisfaction and convenience scores out of 10 for telephone and video consultations $(0=$ very unsatisfied, $10=$ very satisfied)

figure of $50.8 \%$ for telephone consultation patients, suggesting better patient-perceived credibility and communication with video consultations.

\section{- Telephone " Video}

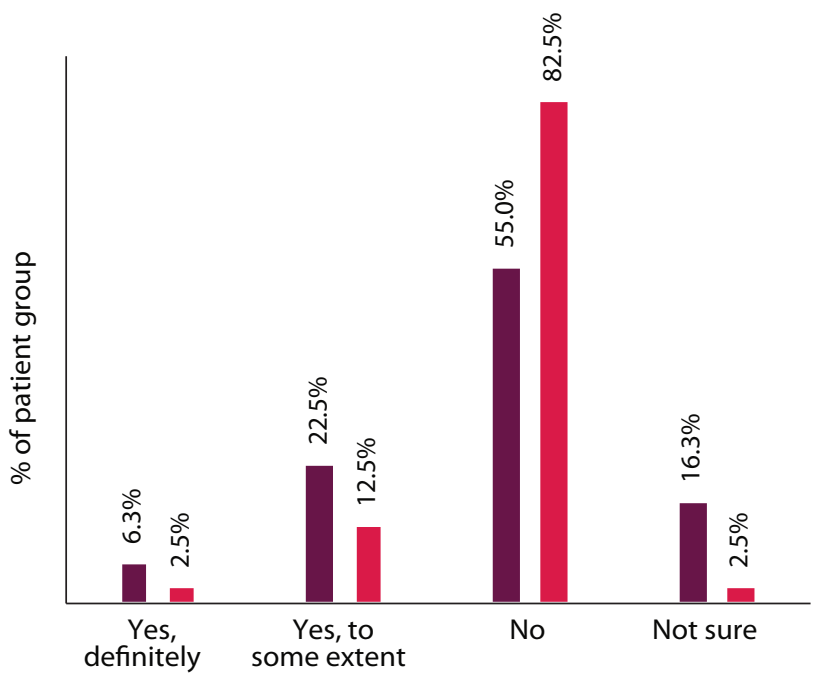

Fig 3. Percentage distribution of patient responses when asked if they felt a face-to-face review would have changed the outcome of the consultation, compared to their telephone or video consultation.

\section{Discussion}

COVID-19 and its effects on the health service will be with us for many months to come. Self-isolation and social distancing demand a redesign of outpatient ophthalmic services, and this provides an opportunity to reconfigure the way we deliver these services to meet current and future demand.

Our experience has shown that teleophthalmology can be incorporated into an existing district general hospital framework with minimal financial investment and minimal disruption to existing frameworks. It was implemented with no new staff requirements, and little in the way of new assets/technology. The lead time from initial discussion to implementation was only 2 weeks. The early involvement of information governance teams meant we were able to develop robust policies that will allow us to continue using the system post-COVID.

A key driver for the successful adoption of teleophthalmology is patient acceptance. Our patient-reported outcome measures demonstrate that patients find both telephone and video consultations beneficial for multiple reasons.

Teleconsultations are substantially more time- and cost-efficient for patients than in-person clinic reviews. Time and expense is saved at all points of the patient journey, and where another individual is required to assist the patient to the hospital this time and cost-saving is effectively doubled. For such reasons, patient satisfaction and convenience have scored highly.

Currently a large proportion of the population are working from home, and therefore likely to be in a confidential space with 


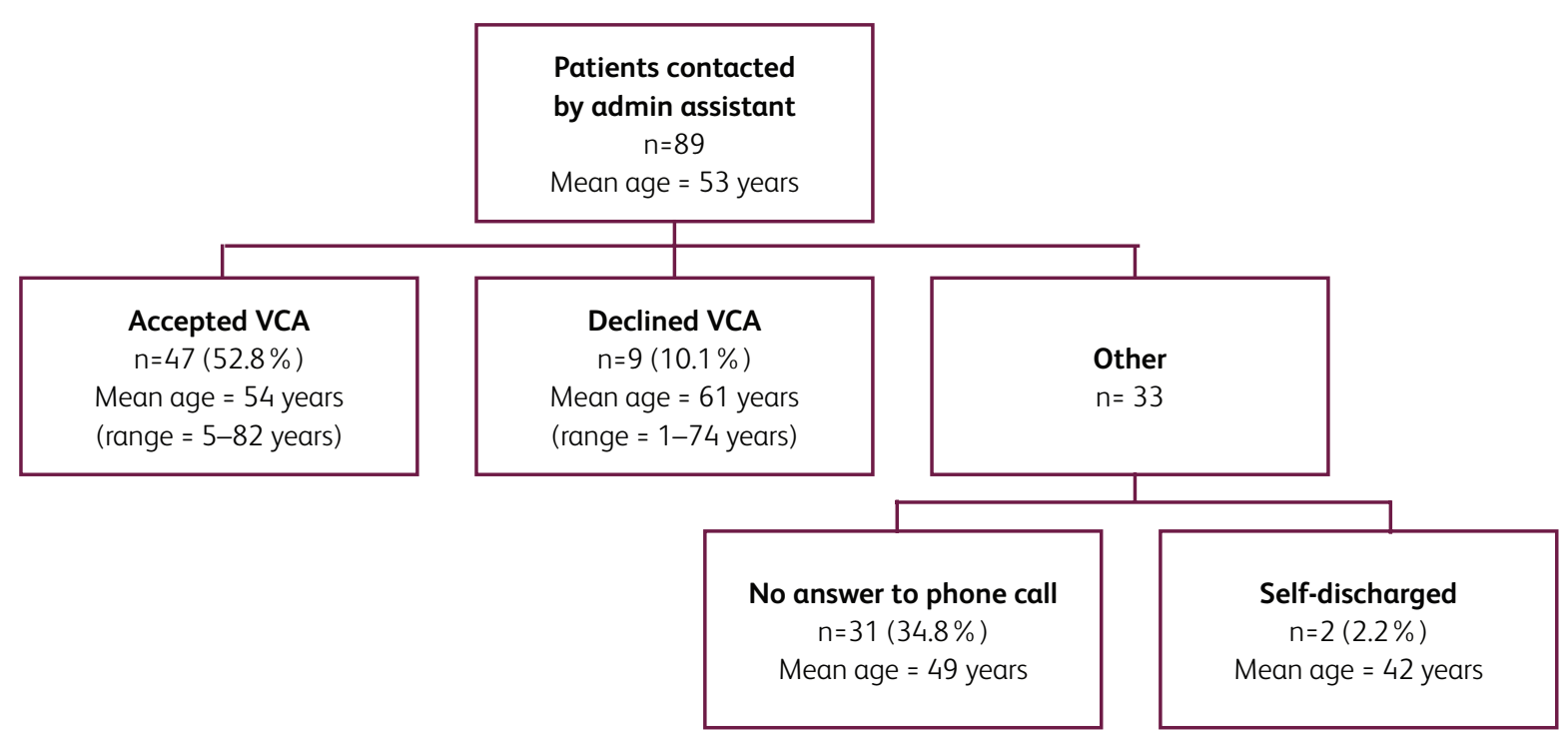

Fig 4. Uptake rate of video consultation appointments (VCA) when patients were contacted by administrative assistant and given a choice between telephone or video consultations.

more time flexibility. This may not be the case in a communal office during a structured working day. Nevertheless, when trying to reach our patients via phone, we found a surprisingly high proportion of people did not answer (Fig 4). Such factors highlight the need for clear, written advice to the patient prior to an appointment with a clearly communicated appointment time such that patients can make themselves available remotely and promptly. We suspect the variability of appointment-related information given to our patients, as reflected in the surveys, was due to department-wide changes to communication strategies during the initial service adaptations of our COVID-19 response.

To allow us to provide truly patient-centred care, it is important to appreciate the benefits of both telephone and video consultations independently. A phone call is easy, requires less technical ability or special equipment and is familiar to all, including the elderly. It serves certain situations very well, for example conveying simple test results or discussing a medication review. Indeed, primary care physicians have been conducting structured telephone clinics for a long time as part of their regular working day. ${ }^{18,19}$ Although telephone consultations are more novel to the secondary care setting, they are serving this purpose just as well. ${ }^{20,21}$

On the other hand, video consultations have the added benefit of conveying visual information. This allows remote examinations to be largely useful and credible, especially for new patients. Patient-clinician interaction via video also helps to establish and maintain rapport. In those cases, where the patient proceeds down a surgical pathway in the future, the familiarity established on the VCA helps build and maintain confidence with the surgical team.

Regarding patient preference of consultation style, our surveys found an expected age bias, with younger patients being more likely to accept teleophthalmology consultations (both telephone and video) and older patients preferring face-to-face appointments. This was occasionally due to lack of access to equipment, but also partially explained by unfamiliarity with technology. We found that telephoning some of the older patients and guiding them through the process of joining a video consultation proved fruitful, and these patients were often pleased with the video consultation and unexpected ease of using the service. We hope this initial time investment will lead to future video appointments feeling more familiar for these individuals.

\section{Beyond COVID-19}

Although the current focus of teleophthalmology is on the response to the COVID-19 pandemic and the need for social distancing, it does also provide the opportunity for a smart redesign of ophthalmic outpatient care.

We envisage a multi-modality clinic encompassing telephone, video and in-person reviews, guided by the clinical and holistic needs of the patient.

Patients requiring technical assistance could be aided by an administrative clerk fulfilling the role of a 'virtual receptionist' or 'virtual assistant' to the clinic. Just as such non-clinical roles are essential to the smooth running of a physical clinic, their digital counterparts will play a vital role in virtual clinic management as demand and capacity for teleophthalmology consultations increase.

By expanding teleophthalmology services into optometry practices, we could further shift the point of care from hospitals to the community. This could serve our patients in many ways, for example providing timely specialist opinion on acute cases that present to the optometrist or providing community-based follow-up clinics with specialist opinion on hand via virtual consultations. Slit-lamp photography and store-and-forward transfer of investigations, such as optical coherence tomography scans and visual fields, will further improve management decisions delivered in the community. Many regions already have accredited optometrists providing community ophthalmology services, and there is huge potential to expand their role in this way. ${ }^{22,23}$

Long-term, these approaches could result in more efficient use of outpatient clinics and limited resources. An added benefit could 
be potential reduction in referral-to-treatment times, and reduced waiting times for patients. This has already been identified as a priority for ophthalmology as part of the NHS Long Term Plan. ${ }^{24}$

Redesigning ophthalmic services at such scale raises questions regarding financial infrastructure. As teleconsultations become integrated into regular outpatient practice, discussions regarding clinic structures and tariffs will evolve. During the current situation, NHS England and NHS Improvement have agreed that video consultations will be reimbursed at the same level as face to face appointments; also, the proposed 2020/21 tariff states that remote activity must have the same unit price as face-to-face attendances. ${ }^{13}$ Currently, most outpatient clinics book 'new' or 'follow-up' appointments and adapt appointment duration and tariff accordingly. Advancements in ophthalmic investigations and data sharing, teamed with remote consultations as described, will allow us to further categorise different appointment types and therefore tariff more appropriately.

In summary, the current pandemic has brought with it an inadvertent opportunity to redefine the way we serve our patients. Teleconsultations have allowed us to keep our patients and clinicians safe whilst providing more efficient ophthalmic care. Our experience in a district general hospital has been a positive team effort and welcomingly adapted by our patients, as described above. We endeavour to continue developing our teleophthalmology services to meet the ongoing needs of our ever-increasing patient population.

\section{Supplementary material}

Additional supplementary material may be found in the online version of this article at www.rcpjournals.org/fhj:

S1 - Telephone survey.

S2 - Video survey.

\section{Acknowledgements}

We would like to acknowledge the following Maidstone and Tunbridge Wells NHS Trust staff for their help and support in procuring and setting up the video calling system promptly and safely: Luke Climpson, transformation programme manager, Gail Spinks, head of information governance, and Miles Scott, chief executive.

\section{References}

1 The NHS long term plan. NHS, 2019. www.longtermplan.nhs.uk/ wp-content/uploads/2019/01/nhs-long-term-plan.pdf [Accessed 11 May 2020].

2 Debnath D. Activity analysis of telemedicine in the UK. Postgrad Med J 2004;80:335-8.

3 NHS Airedale. Telemedicine (Digital Care Hub). Available at www. airedale-trust.nhs.uk/services/telemedicine/ [Accessed 13 May 2020].

4 Scottish Government. Scotland's digital health and care strategy: enabling, connecting and empowering. 2018. www.gov.scot/ publications/scotlands-digital-health-care-strategy-enablingconnecting-empowering/ [Accessed 11 May 2020].

5 Zanaboni P, Wootton R. Adoption of routine telemedicine in Norwegian hospitals: progress over 5 years. BMC Health Serv Res 2016;16:496

6 Donaghy E, Atherton $\mathrm{H}$, Hammersley V et al. Acceptability, benefits, and challenges of video consulting: a qualitative study in primary care. Br J Gen Pract 2019;69:e586-94.
7 Mueller B. Telemedicine arrives in the UK: '10 Years of Change in One Week'. New York Times, 7 April 2020. www.nytimes. com/2020/04/04/world/europe/telemedicine-uk-coronavirus.html.

8 Royal College of Ophthalmologists. COVID-19 clinical guidance and national information. www.rcophth.ac.uk/about/rcophth-covid19-response [Accessed 13 May 2020].

9 Mehrotra A, Chernew M, Linetsky D et al. The impact of the COVID-19 pandemic on outpatient visits: a rebound emerges. Commonwealth Fund, 2020. www.commonwealthfund.org/ publications/2020/apr/impact-covid-19-outpatient-visits [Accessed 10 May 2020].

10 Rayner S, Beaconsfield M, Kennedy C et al. Subspecialty adnexal ophthalmological examination using telemedicine. J Telemed Telecare 2001;7:29-31.

11 Kang S, Thomas PBM, Sim DA et al. Oculoplastic video-based telemedicine consultations: Covid-19 and beyond. Eye 2020;34:1193-5.

12 NHS Digital. Approved video consultation systems. https://digital. nhs.uk/services/future-gp-it-systems-and-services/approvedeconsultation-systems [Accessed 12 May 2020].

13 NHS England. Clinical guide for the management of remote consultations and remote working in secondary care during the coronavirus pandemic. NHSE, 2020. www.england.nhs.uk/coronavirus/ wp-content/uploads/sites/52/2020/03/C0044-Specialty-GuideVirtual-Working-and-Coronavirus-27-March-20.pdf [Accessed 11 May 2020].

14 Denniston AK, Folkard A, Manna E et al. No going back: how patients and professionals are exploring the future of eye services beyond Covid-19. https://blogs.bmj.com/bmj/2020/05/05/nogoing-back-how-patients-and-professionals-are-exploring-thefuture-of-eye-services-beyond-covid-19 [Accessed 13 May 2020].

15 Powell S. Closing the distance. Optometry Today, 22 December 2019. www.aop.org.uk/ot/science-and-vision/technology/ 2019/12/22/closing-the-distance.

16 Serle J. Patients shun video in favour of telephone and messaging. Health Service J, 21 May 2020. www.hsj.co.uk/ service-design/patients-shun-video-in-favour-of-telephone-andmessaging/7027680.article.

17 'Cut-off' over-70s are in the dark about tech. Metro (UK), 15 May 2020.

18 Downes MJ, Mervin MC, Byrnes JM et al. Telephone consultations for general practice: a systematic review. Syst Rev 2017;6:128.

19 Brown A, Armstrong D. Telephone consultations in general practice: an additional or alternative service? Br J Gen Pract 1995;45:673-5.

20 O'Byrne L, Roberts NJ, Partridge MR. Preclinic telephone consultations: an observational cohort study. Clin Med 2012;12:140-5.

21 Roberts N], Partridge MR. Telephone consultations in secondary care. Respir Med. 2007;101:1665-9.

22 Spry PG, Spencer IC, Sparrow JM et al. The Bristol Shared Care Glaucoma Study: reliability of community optometric and hospital eye service test measures. Br ] Ophthalmol 1999;83:707-12.

23 Roberts HW, Rughani K, Syam P et al. The Peterborough scheme for community specialist optometrists in glaucoma: results of 4 years of a two-tiered community-based assessment and follow-up service. Curr Eye Res 2015;40:690-6.

24 Elective Care Transformation Programme. Transforming elective care services: Ophthalmology. NHS England, 2019. www.england. nhs.uk/wp-content/uploads/2019/01/ophthalmology-electivecare-handbook-v1.1.pdf [Accessed 27 May 2020].

Address for correspondence: Mr Sarju Athwal, Eye, Ear and Mouth Unit, Maidstone Hospital, Hermitage Lane, Maidstone, Kent ME16 9QQ, UK.

Email: sarju.athwal@nhs.net 